\title{
THE SCHWARZ LEMMA AND ITS APPLICATION AT A BOUNDARY POINT
}

\author{
MoOnja JEOng
}

\begin{abstract}
In this note we study the Schwarz lemma and inequalities for some holomorphic functions on the unit disc. Also, we obtain the inequality of the derivative of holomorphic maps at a boundary point of the unit disc and find a holomorphic map to satisfy the equality.
\end{abstract}

\section{INTRODUCTION}

One of the very basic theorems in complex analysis is the following Schwarz lemma [4].

Theorem 1.1 (Schwarz Lemma). Let $f$ be a holomorphic function of the open unit disc $U=\{z \in \mathbb{C}:|z|<1\}$ into $U$ with $f(0)=0$. Then $\left|f^{\prime}(0)\right| \leq 1$ and $|f(z)| \leq|z|$ for all $z \in U$ with the equality only for $f(z)=e^{i \theta} z$ with real $\theta$.

If, in addition, $f$ has multiple zeroes at $z=0$, then the Schwarz lemma results in the following (see [7]).

Corollary 1.2. Let $f$ be a holomorphic function of $U$ into $U$ with $f(0)=f^{\prime}(0)=$ $\cdots=f^{(n-1)}(0)=0$. Then $|f(z)| \leq|z|^{n}$ for all $|z|<1$ with the equality only for $f(z)=e^{i \theta} z^{n}$ with real $\theta$.

More generally, the Schwarz lemma can be applied to a function with the information $f(\alpha)=\beta$ for some $\alpha, \beta$ with $|\alpha|<1,|\beta|<1$ instead of $f(0)=0$ and it is called the Schwarz-Pick lemma [2].

Corollary 1.3 (Schwarz-Pick Lemma). Let $f$ be a holomorphic function of $U$ into $U$ with $f(\alpha)=\beta$ for some $\alpha, \beta$ with $|\alpha|<1,|\beta|<1$. Then

Received by the editors May 31, 2014. Accepted July 15, 2014.

2010 Mathematics Subject Classification. Primary: 30C80, Secondary: 30C35.

Key words and phrases. Schwarz lemma, boundary point, unit disc, holomorphic map.

(C) 2014 Korean Soc. Math. Educ. 


$$
\left|f^{\prime}(\alpha)\right| \leq \frac{1-|\beta|^{2}}{1-|\alpha|^{2}}
$$

for $|\alpha|<1$.

If $f$ in Corollary 1.3 fixes $\alpha$, then $\left|f^{\prime}(\alpha)\right| \leq 1$. Note that the equality in the above corollary holds only for Möbius transformation mapping the open unit disc into itself.

The Schwarz lemma looks a simple result, but it is highly influential in the function theory of the complex analysis. It is used to get properties of holomorphic functions of the unit disc into itself at a boundary point of the unit disc. For historical background about the Schwarz lemma and its applications on the boundary of the unit disc, we refer to Boas [1]. In [3], we find a holomorphic self map defined on the closed unit disc with fixed points only on the boundary of the unit disc.

Now, our concern is for holomorphic functions mapping the unit disc into itself at a boundary point of the unit disc. From the Schwarz lemma, it is known that if a holomorphic function $f$ of the unit disc into itself with $f(0)=0$ extends continuously to a boundary point $z_{0}$ with $\left|z_{0}\right|=1,\left|f\left(z_{0}\right)\right|=1$, and $f^{\prime}\left(z_{0}\right)$ exists, then $\left|f^{\prime}\left(z_{0}\right)\right| \geq 1$. The following theorem called the boundary Schwarz lemma can be found in [6].

Theorem 1.4 (The boundary Schwarz Lemma). Let $f$ be a holomorphic function of $U$ into $U$ with $f(0)=0$. Assume that for some point $z_{0}$ with $\left|z_{0}\right|=1, f$ extends continuously to $z_{0},\left|f\left(z_{0}\right)\right|=1$, and $f^{\prime}\left(z_{0}\right)$ exists. Then

$$
\left|f^{\prime}\left(z_{0}\right)\right| \geq \frac{2}{1+\left|f^{\prime}(0)\right|}
$$

and hence

$$
\left|f^{\prime}\left(z_{0}\right)\right| \geq 1 .
$$

The equality in (1.2) holds if and only if $f(z)=z e^{i \theta}$ for some real $\theta$.

If, in addition, the function $f$ has the property $f(0)=f^{\prime}(0)=\cdots=f^{(n-1)}(0)=$ $0, n \in \mathbb{N}$, then

$$
\left|f^{\prime}\left(z_{0}\right)\right| \geq n \text {. }
$$

The equality in (1.3) holds if and only if $f(z)=z^{n} e^{i \theta}$ for some real $\theta$.

Remark 1.5. Under the same hypothesis as in Theorem 1.4 except $f(0)=0$, Osserman [6] showed that the following inequality

$$
\left|f^{\prime}\left(z_{0}\right)\right| \geq \frac{2}{1+\left|F^{\prime}(0)\right|} \frac{1-|f(0)|}{1+|f(0)|}
$$


holds where $F(z)=\frac{f(z)-f(0)}{1-\bar{f}(0) f(z)}$ instead of the inequality (1.1).

The assumption in Theorem 1.4 that $f$ extends continuously to $z_{0}$ with $\left|z_{0}\right|=1$, $\left|f\left(z_{0}\right)\right|=1$, and $f^{\prime}\left(z_{0}\right)$ exists can be changed to the assumption that $f$ has a radial limit $w_{0}$ at $z_{0}$ with $\left|z_{0}\right|=1,\left|w_{0}\right|=1, f$ has a radial derivative at $z_{0}$. (see [6]).

Recently, Örnek [5] proved the following inequality at a boundary point of the unit disc.

Theorem 1.6. Let $f$ be a holomorphic function in $U$ with $f(0)=1$ and $|f(z)-\epsilon|<\epsilon$ for $|z|<1$ and $1 / 2<\epsilon \leq 1$. If for some $z_{0}$ with $\left|z_{0}\right|=1$, $f$ has an angular limit $f\left(z_{0}\right)$ at $z_{0}, f\left(z_{0}\right)=2 \epsilon$, then

$$
\left|f^{\prime}\left(z_{0}\right)\right| \geq \epsilon(2 \epsilon-1) .
$$

Moreover, the equality in (1.5) holds if and only if

$$
f(z)=\epsilon \frac{1+z e^{i \theta}}{\epsilon+(1-\epsilon) z e^{i \theta}}
$$

for a real $\theta$.

In this paper, we show some inequalities at a boundary point for different form of holomorphic functions and find the condition for equality.

\section{The Schwarz Lemma and its Application at a Boundary Point}

The Schwarz lemma means that any holomorphic function of the unit disc into itself with zero fixed maps each disc centered at zero into a smaller one. Moreover it maps each disc centered at zero into a strictly smaller one if it is not a rotation. From now on, more generally we consider a holomorphic function with zero not fixed. Örnek [5] considered a holomorphic function $f$ on $U$ with $f(0)=1,|f(z)-\epsilon|<\epsilon$ where $\epsilon>1 / 2$. We consider a different form of holomorphic functions and get the following proposition by the similar method.

Proposition 2.1. Let $f$ be a holomorphic function on $U$ satisfying $|f(z)-1|<1$ with $f(0)=a$ where $0<a<2$. Then, $f$ satisfies the inequality

$$
|f(z)| \leq \frac{a(1+|z|)}{1-|(1-a) z|}
$$

for $|z|<1$. Moreover,

$$
\left|f^{\prime}(0)\right| \leq a(2-a)
$$


The equality in (2.1) for some nonzero $z \in U$ or in (2.2) holds if and only if

$$
f(z)=\frac{a\left(1+z e^{i \theta}\right)}{1-(1-a) z e^{i \theta}}
$$

for some real $\theta$

Proof. Let $g(z)=f(z)-1$ and let

$$
w(z)=\frac{g(z)-g(0)}{1-\overline{g(0)} g(z)}
$$

for $z \in U$.

Then $g$ and $w$ are holomorphic functions on $U$ with $|g(z)|<1$ and $|w(z)|<1$ for $|z|<1$ and $w(0)=0$. Hence $w$ satisfies the condition for the Schwarz lemma.

By the Schwarz lemma, $|w(z)| \leq|z|$ for $|z|<1$. Hence,

$$
|w(z)|=\left|\frac{f(z)-a}{1-(a-1)\{f(z)-1\}}\right| \leq|z| .
$$

It implies that

$$
\begin{aligned}
|f(z)|-a \leq|f(z)-a| & \leq|z||1-(a-1) f(z)+(a-1)| \\
& \leq|z|\{|(1-a) f(z)|+a\} .
\end{aligned}
$$

Therefore, we have the inequality (2.1).

On the other hand, the facts that

$$
w^{\prime}(z)=\frac{f^{\prime}(z)\{1-(a-1)(f(z)-1)\}+(f(z)-a)(a-1) f^{\prime}(z)}{\{1-(a-1)(f(z)-1)\}^{2}}
$$

and $\left|w^{\prime}(0)\right| \leq 1$ by the Schwarz lemma induce that

$$
\left|w^{\prime}(0)\right|=\frac{\left|f^{\prime}(0)\right|\left\{1-(a-1)^{2}\right\}}{\left\{1-(a-1)^{2}\right\}^{2}} \leq 1 .
$$

Hence, $\left|f^{\prime}(0)\right| \leq 1-(a-1)^{2}=a(2-a)$.

The equality in (2.1) for some nonzero $z \in U$ or in (2.2) holds if and only if

$$
w(z)=\frac{f(z)-a}{1-(a-1)\{f(z)-1\}}=z e^{i \theta},
$$

that is,

$$
f(z)=\frac{a\left(1+z e^{i \theta}\right)}{1-(1-a) z e^{i \theta}}
$$

for some real $\theta$. 
Remark 2.2. From the formula (2.5),

$$
\begin{gathered}
\qquad|f(z)-a|=|f(z)|-a \\
\text { and }|(1-a) f(z)+a|=|(1-a) f(z)|+a
\end{gathered}
$$

should be satisfied at some nonzero point $z \in U$ that the equality in (2.1) holds, i.e., $0<a<1$ and $f(z)$ is real with $a<f(z)<2$ at the above nonzero point $z \in U$.

Therefore, if $f$ is the function in $(2.3)$ and $0<a<1$, then at some nonzero point $z \in U$ satisfying $a<f(z)<2$ the equality in (2.1) holds.

Theorem 2.3. Let $f$ be a holomorphic function on $U$ satisfying $|f(z)-1|<1$ with $f(0)=a$ where $0<a<2$. Assume that for some $z_{0}$ with $\left|z_{0}\right|=1, f$ extends continuously to $z_{0}, f\left(z_{0}\right)=2$, and $f^{\prime}\left(z_{0}\right)$ exists. Then

$$
\left|f^{\prime}\left(z_{0}\right)\right| \geq \frac{2-a}{a}
$$

The equality in (2.6) holds if and only if

$$
f(z)=\frac{a\left(1+z e^{i \theta}\right)}{1-(1-a) z e^{i \theta}}
$$

where some real $\theta$ satisfies that $e^{i \theta}=1 / z_{0}$.

Proof. Let $w$ be the function in (2.4). Then $w^{\prime}(z)$ satisfies that

$$
\begin{aligned}
w^{\prime}\left(z_{0}\right) & =\frac{f^{\prime}\left(z_{0}\right)\{1-(a-1)\}+(2-a)(a-1) f^{\prime}\left(z_{0}\right)}{\{1-(a-1)\}^{2}} \\
& =f^{\prime}\left(z_{0}\right) \frac{(2-a)(1+a-1)}{(2-a)^{2}} \\
& =f^{\prime}\left(z_{0}\right) \frac{a}{2-a} .
\end{aligned}
$$

The inequality $\left|w^{\prime}\left(z_{0}\right)\right| \geq 1$ in (1.2) implies that

$$
\left|f^{\prime}\left(z_{0}\right)\right| \geq \frac{2-a}{a} .
$$

If $\left|f^{\prime}\left(z_{0}\right)\right|=\frac{2-a}{a}$, then $\left|w^{\prime}\left(z_{0}\right)\right|=1$ and so by Theorem 1.4, $w(z)=z e^{i \theta}$ for some real $\theta$. It means that

$$
f(z)=\frac{a\left(1+z e^{i \theta}\right)}{1-(1-a) z e^{i \theta}}
$$

for some real $\theta$. By the condition $f\left(z_{0}\right)=2$,

$$
a\left(1+z_{0} e^{i \theta}\right)=2\left\{1-(1-a) z_{0} e^{i \theta}\right\}
$$

Hence, $z_{0} e^{i \theta}=1$ and so $\theta$ satisfies that $e^{i \theta}=1 / z_{0}$. Conversely, for the given function 


$$
f(z)=\frac{a\left(1+z e^{i \theta}\right)}{1-(1-a) z e^{i \theta}}
$$

where $\theta$ satisfies that $e^{i \theta}=1 / z_{0}$, the equality in (2.6) holds.

Remark 2.4. The inequalities in Proposition 2.1 and Theorem 2.3 with $a=1$ coincide with the inequalities in Theorem 1.6 and a theorem in Örnek [5] with $\epsilon=1$.

Now, we consider a holomorphic function $f$ with $f(0)-a=f^{\prime}(0)=\cdots=$ $f^{(n-1)}(0)=0$. A function given by

$$
f(z)=a+c_{n} z^{n}+c_{n+1} z^{n+1}+\cdots, n \in \mathbb{N}
$$

with $c_{n} \neq 0$, is such a holomorphic function. If we change the role $\left|w^{\prime}\left(z_{0}\right)\right| \geq 1$ by $\left|w^{\prime}\left(z_{0}\right)\right| \geq n$ in the proof of Theorem 2.3, then the following corollary holds.

Corollary 2.5. Let $f$ be a holomorphic function defined on $U$ by

$$
f(z)=a+c_{n} z^{n}+c_{n+1} z^{n+1}+\cdots, n \geq 1
$$

satisfying $|f(z)-1|<1$ on $U$ where $0<a<2$ and $c_{n} \neq 0$. Assume that for some $z_{0}$ with $\left|z_{0}\right|=1$, $f$ extends continuously to $z_{0}, f\left(z_{0}\right)=2$, and $f^{\prime}\left(z_{0}\right)$ exists. Then

$$
\left|f^{\prime}\left(z_{0}\right)\right| \geq n \frac{(2-a)}{a} .
$$

The equality in (2.8) holds if and only if

$$
f(z)=\frac{a\left(1+z^{n} e^{i \theta}\right)}{1+(a-1) z^{n} e^{i \theta}}
$$

where $\theta$ satisfies $e^{i \theta}=1 / z_{0}^{n}$.

Proof. By using the formula of $w$ in (2.4),

$$
\begin{aligned}
w(z) & =\frac{(f(z)-1)-(f(0)-1)}{1-(\overline{f(0)-1})(f(z)-1)} \\
& =\frac{c_{n} z^{n}+c_{n+1} z^{n+1}+\cdots}{1-(a-1)^{2}-(a-1)\left(c_{n} z^{n}+c_{n+1} z^{n+1}+\cdots\right)} \\
& =b_{n} z^{n}+b_{n+1} z^{n+1}+\cdots
\end{aligned}
$$

where $b_{n}, b_{n+1}, \cdots \in \mathbb{C}$ and $b_{n}=c_{n} /\left\{1-(a-1)^{2}\right\} \neq 0$.

Hence, $w(0)=w^{\prime}(0)=\cdots=w^{(n-1)}(0)=0$. By Theorem 1.4 and $(2.7)$,

$$
n \leq\left|w^{\prime}\left(z_{0}\right)\right|=\left|f^{\prime}\left(z_{0}\right)\right| \frac{a}{2-a}
$$

holds and we get (2.8). 
The following theorem provides a refined inequality at a boundary point $z_{0}$ than Theorem 2.3. If we apply the inequality (2.2), we find that the following inequality (2.10) implies the inequality (2.6).

Theorem 2.6. Let $f$ be a holomorphic function on $U$ satisfying $|f(z)-1|<1$ with $f(0)=a$ where $0<a<2$. Assume that for some $z_{0}$ with $\left|z_{0}\right|=1, f$ extends continuously to $z_{0}, f\left(z_{0}\right)=2$, and $f^{\prime}\left(z_{0}\right)$ exists. Then

$$
\left|f^{\prime}\left(z_{0}\right)\right| \geq \frac{2(2-a)^{2}}{a(2-a)+\left|f^{\prime}(0)\right|}
$$

The equality in (2.10) holds for the function

$$
f(z)=\frac{1+2 b z+z^{2}}{a\{2-a+(b+a-1) z\}}
$$

with $z_{0}=1$ where $b=\frac{\left|f^{\prime}(0)\right|}{a(2-a)}$ and $a=1$, i.e.,

$$
f(z)=\frac{1+2 b z+z^{2}}{1+b z}
$$

with $z_{0}=1$ where $0 \leq b=\left|f^{\prime}(0)\right| \leq 1$.

Proof. Let $w$ be the function in (2.4). By applying the inequality (1.1) to $w^{\prime}(z)$ and the equation (2.7),

$$
\frac{2}{1+\left|w^{\prime}(0)\right|} \leq\left|w^{\prime}\left(z_{0}\right)\right|=\left|f^{\prime}\left(z_{0}\right)\right| \frac{a}{2-a} .
$$

Since,

$$
w^{\prime}(0)=\frac{f^{\prime}(0)\left\{1-(a-1)^{2}\right\}}{\left\{1-(a-1)^{2}\right\}^{2}}=\frac{f^{\prime}(0)}{1-(a-1)^{2}}=\frac{f^{\prime}(0)}{a(2-a)}
$$

we have

$$
\frac{2}{1+\frac{\left|f^{\prime}(0)\right|}{a(2-a)}} \leq\left|f^{\prime}\left(z_{0}\right)\right| \frac{a}{2-a} .
$$

Hence,

$$
\left|f^{\prime}\left(z_{0}\right)\right| \geq \frac{2-a}{a} \frac{2 a(2-a)}{a(2-a)+\left|f^{\prime}(0)\right|}=\frac{2(2-a)^{2}}{a(2-a)+\left|f^{\prime}(0)\right|} .
$$

For the equality in (2.10), choose arbitrary $b$ satisfying $0 \leq b \leq 1$ and let

$$
f(z)=\frac{1+2 b z+z^{2}}{a\{2-a+(b+a-1) z\}} .
$$

Then,

$$
f^{\prime}(z)=\frac{(2 b+2 z)\{2-a+(b+a-1) z\}-\left(1+2 b z+z^{2}\right)(b+a-1)}{a\{2-a+(b+a-1) z\}^{2}}
$$


and it implies that

$$
f^{\prime}(1)=\frac{2(2-a)}{a(b+1)} .
$$

Therefore the equality in (2.10) holds at $z_{0}=1$ where $b=\frac{\left|f^{\prime}(0)\right|}{a(2-a)}$.

On the other hand,

$$
f(1)=\frac{1+2 b+1}{a(2-a+b+a-1)}=\frac{2}{a} .
$$

In order to satisfy that $f(1)=2$, the condition $a=1$ should be satisfied. So,

$$
f(z)=\frac{1+2 b z+z^{2}}{1+b z}
$$

where $b=\frac{\left|f^{\prime}(0)\right|}{a(2-a)}=\left|f^{\prime}(0)\right| \leq 1$.

Remark 2.7. The function $f$ in (2.11) can be represented by the following interesting Mclaurin series at zero.

$$
\begin{aligned}
f(z) & =\frac{1+2 b z+z^{2}}{1+b z} \\
& =1+b z+\left(1-b^{2}\right) z^{2} \frac{1}{1+b z} \\
& =1+b z+\left(1-b^{2}\right) z^{2}\left\{1-b z+(b z)^{2}-(b z)^{3}+\cdots\right\} .
\end{aligned}
$$

where $0 \leq b=\left|f^{\prime}(0)\right|=f^{\prime}(0) \leq 1$ for $|z|<1$.

For example, if $b=0$, then $f(z)=1+z^{2}$.

If $b=1$, then $f(z)=1+z$.

If $b=\frac{1}{2}$, then $f(z)=\frac{1+z+z^{2}}{1+\frac{1}{2} z}=1+\frac{1}{2} z+\frac{3}{4} z^{2}+\cdots$.

\section{REFERENCES}

1. H.P. Boas: Julius and Julia: Mastering the Art of the Schwarz Lemma. Amer. Math. Monthly 117 (2010), 770-785.

2. R. Greene \& S. Krantz: Function theory of one complex variable, Graduate studies on Mathematics Vol. 40. Amer. Math. Soc., Providence, 2002.

3. M. Jeong: The Schwarz lemma and boundary fixed points. J. Korean. Soc. Math. Educ. Ser. B: Pure Appl. Math. 18 (2011), no. 3, 275-284.

4. Z. Nehari: Conformal Mapping. Dover publications, Inc., New York, 1952.

5. B. Örnek: Scharpened forms of the Schwarz lemma on the boundary. Bull. Korean Math. Soc. 50 (2013), 2053-2059. 
6. R. Osserman: A Sharp Schwarz Inequality on the boundary. Proc. Amer. Math. Soc. 128 (2000), 3513-3517.

7. H. Silverman: Complex Variables. Houghton Mifflin, 1975.

Department of Mathematics, The University of Suwon, Gyeonggi-do 445-743, Korea

Email address: mjeong@suwon.ac.kr 